\title{
Effect of Structure and Properties of Magnetic Material on Diffraction Efficiency of Magnetophotonic Crystal Media for Magnetic Volumetric Holography
}

\author{
Ryosuke Isogai, Taichi Goto, Hiroyuki Takagi, Yuichi Nakamura, Pang Boey Lim, and Mitsuteru Inoue \\ Toyohashi University of Technology, 1-1 Hibarigaoka, Tempaku, Toyohashi, Aichi 441-8580, Japan
}

\begin{abstract}
Magnetic holography is a rewritable holographic technology with magnetic media. We reported that magnetophotonic crystal (MPC) media are effective to bring high diffraction efficiency because of an enhancement of Faraday rotation angle and deep magnetic fringes. In this paper, we investigated the MPC media with more than 2-pair Bragg mirrors, which may show much higher efficiency due to their enhanced Faraday rotation angle. However, the intensity of diffracted beam of such the MPCs decreased because of the low transmittance due to the absorption of magnetic material. The relationship between the optical properties of magnetic material in MPCs and the diffraction efficiencies were investigated. High optical efficiencies will be achieved by using the magnetic material with high magneto-optical figure of merit such as bismuth substituted gadolinium iron garnet.
\end{abstract}

Key words: magnetic hologram, magnetophotonic crystal, diffraction efficiency, magneto-optical recording

\section{Introduction}

Demands on a high-capacity data storage system have been increasing in information era, and the development of such the storage device is an urgent task. Hologram memory is a strong candidate for the novel storage technology with high recording density and high transfer rate ${ }^{1)}$. We have studied a magnetic hologram, which is the hologram recorded in a magnetic film as a distribution of magnetization directions for a rewritable hologram memory ${ }^{2}$. Transparent magnetic garnets are used for a medium of volumetric magnetic holograms. For instance, $\mathrm{Bi}_{1.3} \mathrm{Dy}_{0.85} \mathrm{Y}_{0.85} \mathrm{Fe}_{3.8} \mathrm{Al}_{1.2} \mathrm{O}_{12}$ (BiDyAlYIG) is a transparent magneto-optical (MO) material providing large Faraday rotation angle in the visible range ${ }^{3)}$. We used this BiDyAlYIG film for the magnetic holograms ${ }^{4)-5)}$, while the diffraction efficiency of the monolayer film is not sufficient to apply to storage devices.

Recently, we reported that magnetophotonic crystal (MPC) media were effective to show high diffraction efficiencies because of the enhancement of Faraday rotation angle and deep magnetic fringes ${ }^{6}$. The MPC structure is a stacking layered structure where the magnetic film is sandwiched between two Bragg mirrors $(\mathrm{BMs})$. This structure acts as a MO micro cavity and enhances the Faraday rotation angle ${ }^{7)-9)}$. In the previous work $^{6)}$, we investigated the enhancement of diffraction efficiency for only low-Q BiDyAlYIG MPCs with small repeated pairs (e.g. 1 or 2 pairs) in the BMs and only with the narrow range of the garnet thickness around $3.88 \mu \mathrm{m}$. High-Q MPC media are expected to show much higher diffraction efficiency because of their high Faraday rotation angle. In this work, we investigated the effect of high-Q MPC media with more than 2-pair $\mathrm{BMs}$ on the diffraction efficiency by numerical calculations.

\section{Calculation method}

The structure of the MPC media used in the simulation was substrate / $\left(\mathrm{Ta}_{2} \mathrm{O}_{5} / \mathrm{SiO}_{2}\right)^{r} / \mathrm{BiDyAlYIG} /$ $\left(\mathrm{SiO}_{2} / \mathrm{Ta}_{2} \mathrm{O}_{5}\right)^{r}$. The thickness of the amorphous $\mathrm{SiO}_{2}$ and $\mathrm{Ta}_{2} \mathrm{O}_{5}$ layers were $91.7 \mathrm{~nm}$ and $61.6 \mathrm{~nm}$, respectively, according to the design of BMs, $\lambda / 4 n$, where $\lambda=532 \mathrm{~nm}$ is the center wavelength of the photonic band gap, and $n$ is the refractive index of each material. The material parameters used in the simulation are shown in Table 1. The diffraction efficiencies of the MPC media were evaluated by finite element method (COMSOL Multiphysics $4.3 \mathrm{a}$ ) with varying the number of repeated pairs in the BMs, $r$, and the thickness of BiDyAlYIG layer, tYIG. A writing power density was also varied in the calculations because it affects the depth of magnetic fringes and there is an optimum power density for each structure $^{10)}$. In this paper, the MPC structure with the $\mathrm{BMs}$ where the $r$ pairs of layers are located is hereafter referred to " $r$-pair MPC".

The simulation model was based on a two-beam interferometer, where a signal beam and a reference beam with the wavelength of $\lambda=532 \mathrm{~nm}$ were interfered on a medium to record an interference pattern. Then only the reference beam was incident to the medium and the hologram was reconstructed via diffraction phenomena. The calculation process is as follows. First the interference between the signal and the reference beams was calculated by electromagnetic simulation. The distribution of dissipation energy density, which is

Table 1 Material parameters in the simulations ${ }^{a}$

\begin{tabular}{cccc}
\hline Material & $n$ & $\kappa$ & $\theta_{\mathrm{F}}(\mathrm{deg} / \mu \mathrm{m})$ \\
\hline $\mathrm{BiDyAlYIG}$ & 2.09 & 0.0186 & 2.26 \\
$\mathrm{SiO}_{2}$ & 1.45 & 0 & - \\
$\mathrm{Ta}_{2} \mathrm{O}_{5}$ & 2.16 & 0 & - \\
\hline
\end{tabular}

aRefractive index $n$, extinction coefficient $\kappa$, and specific Faraday rotation angle $\theta_{\mathrm{F}}$. 
the energy of light absorbed in the garnet film, was calculated. In the next step, the thermal analysis was carried out by solving thermal diffusion equation using this dissipation energy as the heat source for 50 psec, and the time development of the temperature distribution was calculated during laser radiation and following cooling process. Based on this temperature distribution, the shape of magnetic fringes was determined assuming that the magnetization was reversed where the temperature surpasses the Curie temperature of the garnet $\left(150{ }^{\circ} \mathrm{C}\right)$. To reconstruct the hologram, only the reference beam was irradiated to the determined magnetic fringe. In this calculation, we expressed the magnetic reversal as the sign inversion of the off-diagonal elements in the permittivity tensor of the garnet layer. The electromagnetic simulation was performed and the diffraction efficiency $\eta$, was derived from the simulation by the following equation ${ }^{11}$,

$$
\eta=\frac{I_{1}}{I_{0}+I_{1}} \times 100 \quad(\%),
$$

where $I_{0}$ and $I_{1}$ are the intensities of the transmitted beam and the 1st-order diffracted beam, respectively.

\section{Effect of light confinement in cavity on diffraction efficiencies}

Fig. 1 shows the garnet thickness, tYIG, dependence of diffraction efficiency for the monolayer film and the MPC media. In the MPCs the maximal peaks of diffraction efficiency exist at the resonant thickness $d_{\text {res }}$,

$$
d_{\text {res }}=\frac{m \lambda}{2 n \cos \varphi},
$$

where $m$ is an integer, $\lambda$ is the wavelength, $n$ is the refractive index of the garnet, and $\varphi$ is the angle between the beam and the normal to the surface of the medium, respectively. At the resonant thickness, the system satisfies the Fabry-Pérot resonant condition, and the Faraday rotation angle is enhanced. Fig. 2 shows the dependence of the repeated pairs in BMs, $r$, on the maximal Faraday rotation angle and diffraction efficiency. Note that $t_{\mathrm{YIG}}$ for each structure was set to be $d_{\text {res }}$ in Fig. 2, and we compare the effect of structures at the resonant condition. As shown in Fig. 2, the large- $r$ MPCs, which meant MPC structures with high confinement of light, resulted in large rotation angles and consequently high diffraction efficiencies. This may suggest that the large- $r$ MPCs are favorable for hologram media. However, when we focused on the diffracted beam intensity $I_{1}$, which was equivalent to the brightness of the reconstructed beam, another issue was found. The $r$ dependence of $I_{1}$ is shown in Fig. 3. The maximum intensity was obtained at $r=1$, then the intensity decreased with increasing $r$. This behavior is different from the $r$ dependence of diffraction efficiency. A reason for having the maximum value in the $r$ dependence of $I_{1}$ is the reduction of transmittance of the MPC structures shown in Fig. 3. The transmittance of the large- $r$ MPCs is significantly low, and this means

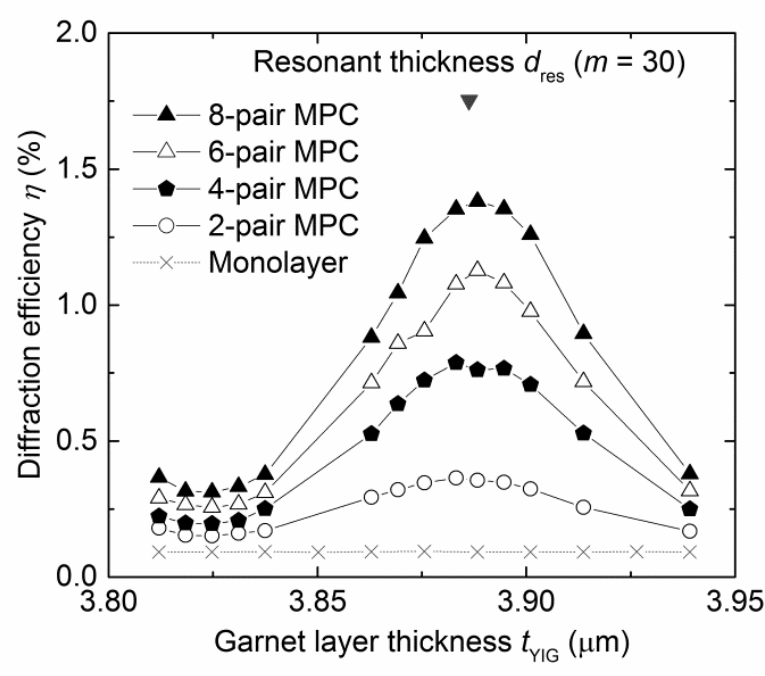

Fig. 1 Garnet layer thickness dependence of the diffraction efficiency of the MPC media.

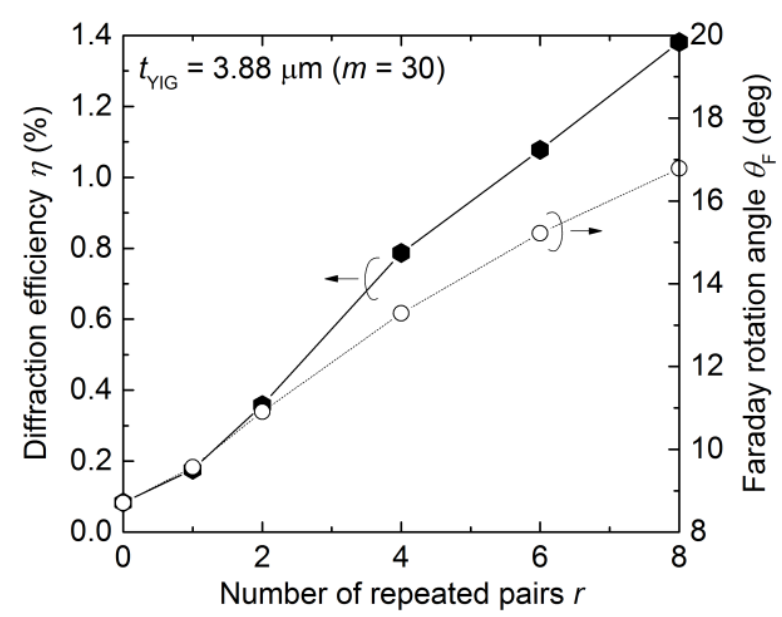

Fig. 2 Relationship of diffraction efficiency and rotation angle of the MPC structures at $m=30$ with the repeated pairs in BMs.

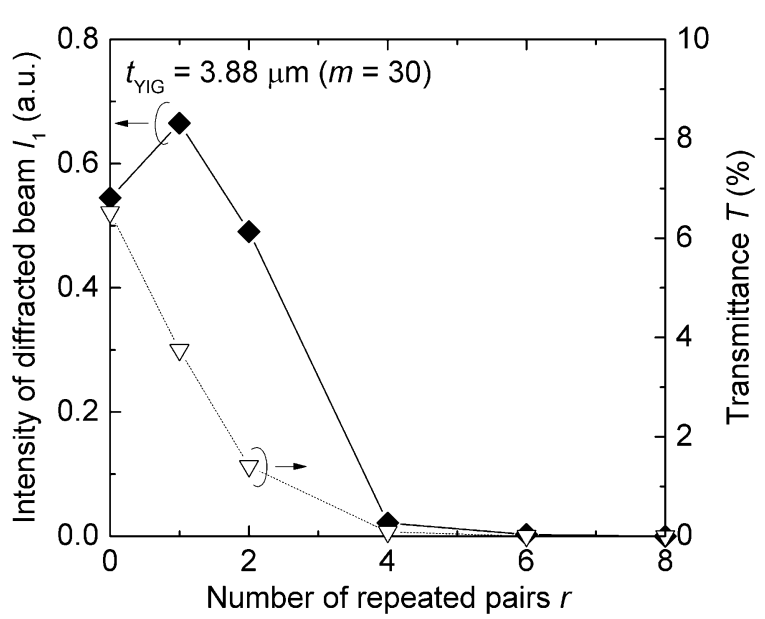

Fig. 3 Relationship of the intensity of diffracted beam and the transmittance with the repeated pairs in BMs. 


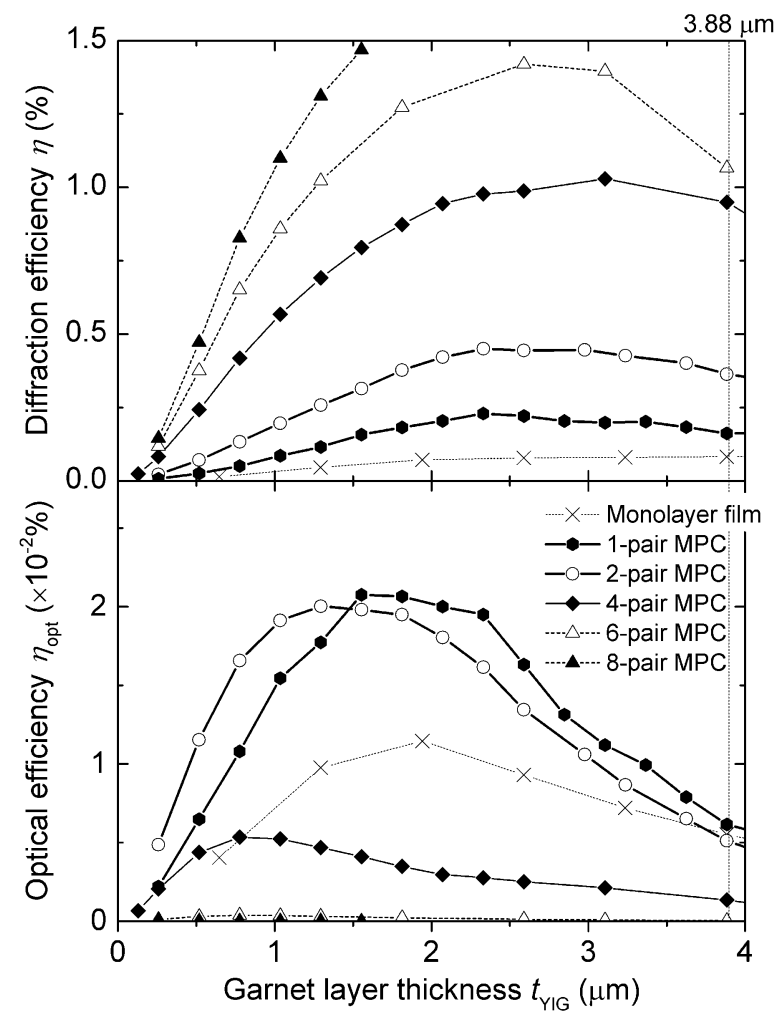

Fig. 4 The diffraction and optical efficiencies for the MPCs with a wide range of $t_{\mathrm{YIG}}(0-4 \mu \mathrm{m})$.

that the amount of light contributing the diffraction is decreased. A reason of this low transmittance in large- $r$ MPCs would be attributed to the absorption in garnet. Since the MPCs are composed of a light absorbable material like magnetic garnet, the light decays during propagation through the garnet layer. In addition, the reflection at the BM surfaces is also increased because optical cavities with an absorbable layer partially act as reflective BMs rather than cavities. This resultant low transmittance caused the low intensity of the diffracted light, which means the medium provides only a dark reconstructed image.

Based on this consideration, since the transmittance is notably depending on the structure and thickness of the medium, an optical efficiency, $\eta_{\text {opt, }}$ is used as a performance index considering the transmittance for the evaluation of MPC media,

$$
\eta_{\text {opt }}=\frac{I_{1}}{I_{\text {in }}} \times 100=T \times \eta \quad(\%),
$$

where $I_{\text {in }}$ is the intensity of the incident beam, and $T$ is the transmittance of the medium, respectively. This index indicates the intensity of the diffracted beam when the incident light power is constant. We evaluated the diffraction efficiency and the optical efficiency of the MPC media in the thickness range of $0-4 \mu \mathrm{m}$ because the transmittance of the structure is sensitive to changes in the thickness. The results are shown in Fig. 4, where all the points are at the resonant thicknesses, $d_{\text {res, }}$, which are the local maximal values of the efficiencies as shown in Fig. 1. In the diffraction efficiency, since the
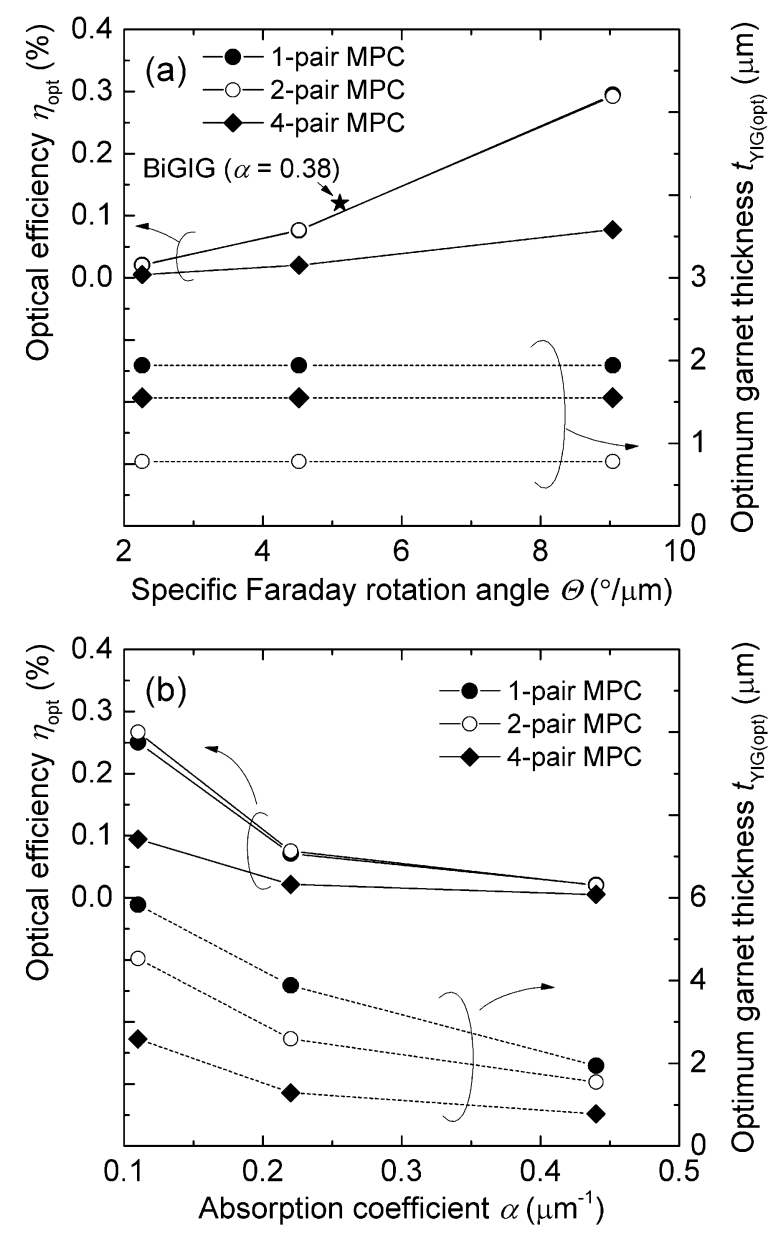

Fig. 5 The dependence of the optical efficiency on the optical properties of garnet material with varying (a) specific rotation angle and (b) absorption coefficient.

effect of transmittance is not included, the large- $r$ MPCs showed the high diffraction efficiency at any thickness because of the strong localization in large- $r$ MPCs that brings the large rotation angle even in low transmittance conditions. Even though the effect of transmittance was not included, the diffraction efficiency showed the maximum value at a certain

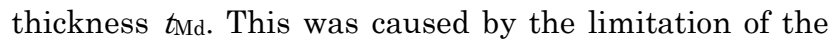
effective magnetic fringe depth in the thick garnet layer ${ }^{10)}$. When $t_{\mathrm{YIG}}$ is smaller than $t_{\mathrm{Md}}$, the thickness of the garnet layer is too thin to form sufficiently deep magnetic fringes. On the other hand, when tyIG exceeds $t_{\mathrm{Md}}$, in addition to the saturation of effective fringe depth, a large absorbance of the thick defect layer in the cavity might weaken the localization of light and may decrease the Faraday rotation angle. So the balance of these factors represents the maxima at $t_{\mathrm{YIG}}=t_{\mathrm{Md}}$ in the relationship between $\eta$ and tyIG.

In contrast to the diffraction efficiency, the optical efficiencies showed the clear maximum value at the certain thickness, $d_{\text {Mopt }}$, determined by both the diffraction efficiency and the transmittance depending on the structure of the media. In the MPC media with 
large number of pairs of $\mathrm{BMs}$, the thickness at maximum optical efficiency, $d$ Mopt, shifted to thinner thickness because the effect of above-described reduction of transmittance became dominant. In this analysis, the maximum optical efficiency of $0.02 \%$ for the 1 -pair and 2-pair MPCs was about twice larger than that of the monolayer film. In contrast, the 4-pair and more- $r$ MPCs showed low efficiencies because of the low transmittance. In the case using BiDyAlYIG as magnetic defect layer in a MPC, the 1-pair MPC provided the highest optical efficiency. However, when another material with large transmittance and/or large Faraday rotation is applied to MPC structures, the dependence of the optical efficiencies on $r$ and tYIG will be changed according to the optical properties of the defect material.

\section{Influence of optical properties of defect layer}

As described in the previous section, the diffraction efficiency and the optical efficiency depend on the properties of magnetic material in the defect layer. To clarify the influence of properties of the magnetic material, we investigated the effect of the optical properties of magnetic material on the optical efficiency; the specific Faraday rotation angle per unit length $\Theta$ and absorption coefficient $\alpha$ of the garnet material were varied, and the effects on the optical efficiency was evaluated. The optical efficiency of the MPC media was evaluated with changing specific Faraday rotation angle, $\Theta$, in the range from 2.26 to 9.04 degree/ $\mu \mathrm{m}$ (factor of 1,2 , and 4 to $\left.\Theta_{\mathrm{YIG}}\right)$ and absorption coefficient, $\alpha$, from 0.44 to $0.11 \mu^{-1}$ (factor of $1,1 / 2$, and $1 / 4$ to $\alpha \mathrm{YIG}$ ), on the basis of the original material parameters of BiDyAlYIG, OrIG $=$ 2.26 degree $/ \mu \mathrm{m}$ and $\alpha \mathrm{YIG}=0.44 \mu \mathrm{m}^{-1}$.

Fig. 5 shows the $\Theta$ and $\alpha$ dependences of the optical efficiency, and the optimal thicknesses of tyIG in each condition are also shown in this figure. All the plots in

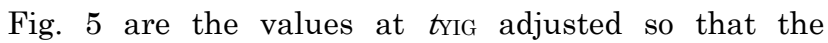
maximum efficiency was obtained. The influence of $\Theta$ was simple; the optical efficiency was proportional to $\Theta^{2}$, and MO films with large Faraday rotation brought high efficiencies at the optimal thickness of tYIG as shown in Fig. 5 (a). In contrast to $\Theta$ value, the absorption coefficient, $\alpha$, strongly affected the optimal structure of MPC media. In the case of $\alpha=0.44 \mu \mathrm{m}^{-1}$, the optical efficiencies were very similar for all MPCs. On the other hand, when the $\alpha$ decreased to $0.11 \mu \mathrm{m}^{-1}$, the optimal thickness increased and the optical efficiency of the 2-pair MPC was the highest. This indicates that the suitable $r$ of MPCs and the thickness of garnet layer should be designed depending on the absorbance of material. For example, when a bismuth substituted gadolinium iron garnet ${ }^{12}$, the MO material with $\alpha \sim 0.38$ $\mu \mathrm{m}^{-1}$ and $\Theta \sim 5.1 \% \mathrm{~m}$ at $532 \mathrm{~nm}$, is applied to the 2-pair MPC, the optical efficiency of $0.12 \%$ is expected from the calculations as shown in Fig. 5 (a). This value is about six times larger than the efficiency of the BiDyAlYIG 2-pair MPC. They mean that the use of magnetic material with a high MO figure of merit $\left.(F O M=\Theta / \alpha)^{13}\right)$ is effective to achieve a high efficiency.

\section{Conclusions}

We evaluated the diffraction efficiencies of the MPC media with several numbers, $r$, of pairs in BMs, which is expected to bring high diffraction efficiency because of their enhanced rotation angle. As expected, the large- $r$ MPC media showed the large Faraday rotation angle and high diffraction efficiency. However the intensity of reconstructed beam was found to be decreased in such the large- $r$ MPCs. The reason for this low-intensity diffracted light was the decrease in transmittance of the structure. For the design of MPC holographic media, we have to consider the balance between the transmittance and diffraction efficiency of the media to achieve high performance.

All magnetic materials have the optical absorption from atoms and ions contributing its magnetism. This suggests that the selection of magnetic material is important. When the bismuth substituted gadolinium iron garnet having the large MO figure of merit is applied, the optical efficiency of $0.12 \%$ is expected and is about 6 times larger than that of the BiDyAlYIG MPC media.

Acknowledgements This work was supported in part by the Grants-in-Aid for Scientific Research (S) 26220902 and Grant-in-Aid for Japan Society for the Promotion of Science (JSPS) Fellows No. 25-8942.

\section{References}

1) H. J. Coufal, D. Psaltis, G. T. Sincerbox, A. M. Glass and M. J. Cardillo: Holographic Data Storage, (Springer, New York, 2000).

2) G. Fan, K. Pennington and J. H. Greiner: J. Appl. Phys., 40, 974 (1969)

3) S. Wittekoek, T. J. A. Popma, J. M. Robertson and P. F. Bongers: Phys. Rev. B, 12, pp.2777-2788 (1975).

4) S. Baek, H. Sakurai, P. B. Lim, A. V. Baryshev, Y. Nakamura, H. Takagi and M. Inoue: IEICE Technical Report, 111, pp.21-25 (2011).

5) Y. Nakamura, H. Takagi, P. B. Lim, M. Inoue: Opt. Exp., 22 pp.16439-16444 (2014).

6) R. Isogai, N. Sagara, T. Goto, Y. Nakamura, P. B. Lim, and M. Inoue: J. Magn. Soc. Jpn., 38, pp.119-122 (2014).

7) M. Inoue, R. Fujikawa, A. V. Baryshev, A. Khanikaev, P. B. Lim, H. Uchida, O. Aktsipetrov, A. Fedyanin, T. Murzina, and A. Granovsky: J. Phys. D, 39, pp.R151-R161 (2006).

8) A. M. Grishin and S. I. Khartsev: J. Magn. Soc. Jpn., 32, pp. 140-145 (2008).

9) M. Levy: J. Appl. Phys., 99, 073104 (2006).

10) Y. Nakamura, H. Takagi, P. B. Lim and M. Inoue: J. Appl. Phys., 116, 103106 (2014).

11) H. Horimai, A. Inoue, T. Miyama, T. Fujii, S. Suzuki, R. Kusaka and T. Tokushima: J. Magn. Soc. Jpn., 8, pp.109-112 (1984) (in Japanese).

12) H.Takeuchi: Jpn. J. Appl. Phys., 14, pp.1903-1910 (1975).

13) J. F. Dillon Jr.: J. Appl. Phys., 39, pp.922-929 (1968).

Received Oct. 20, 2014; Accepted Dec. 26, 2014 